\title{
SYNTHESIS AND STRUCTURAL DETERMINATION OF A NEW CHALCONE 1,5-BIS(3-METHYL-2-THIENYL)PENTA-1,4-DIEN-3-ONE, $\mathrm{C}_{15} \mathrm{H}_{14} \mathrm{OS}_{2}$
}

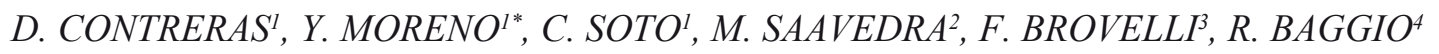 \\ 1_Depto. de Química Analítica e Inorgánica, Facultad de Ciencias Químicas, Universidad de Concepción, Chile \\ 2_Depto. de Fisicoquímica, Facultad de Ciencias Químicas, Universidad de Concepción, Chile \\ 3 Depto. de Ciencias Básicas, Lab. Biotecnología y estudios ambientales, Campus Los Ángeles, Universidad de Concepción, Chile \\ 4_Depto. de Física, Comisión Nacional de Energía Atómica, Buenos Aires, Argentina.
}

(Received: July 14, 2009 - Accepted: September 24, 2009)

\begin{abstract}
This is a structure in a family concerning new polythiophene derivatives. This kind of compounds, a chalcones, has interesting aspects about its intermolecular interactions, of varied type $(\mathrm{C}-\mathrm{H} \cdots \mathrm{O}, \mathrm{C}-\mathrm{H} \cdots \pi, \pi \cdots \pi)$ and strength. In the case of the title compound, $\mathrm{C}_{15} \mathrm{H}_{14} \mathrm{OS}_{2}$, interactions of all three types generate a rather compact, evenly connected 3D structure.
\end{abstract}

\section{INTRODUCTION}

The electronic and optoelectronic applications of conjugated polymers as organic wide-gap semi-conductors have attracted considerable interest in the last decade. In this field, polythiophenes derivatives are among the most studied because of their potential use in organic light-emitting diodes. ${ }^{1-3}$

These polymers (used to prepare electroluminicent devices) are obtained through monomer polymerization via electrochemical deposition on indium tin oxide. ${ }^{4,5}$ The polymeric films obtained show photoluminiscent emission between 660 and $720 \mathrm{~nm}$ and exhibit a turn-on voltage at about 2-4 V. The I-V curves of device have been interpreted by mean tunneling process described by the Fowler-Nordheim theory. ${ }^{5,6}$ The polymeric precursors employed have been a series of heterocyclic $\alpha, \beta$ '-unsaturated ketones containing thiophene rings $4,5,7,8$ similar to the molecule reported in this work and it has been studied and characterized by electrochemical and ab-initio methods., ${ }^{9,10}$

Computational studies proved that the conformation of these molecules are the result of the interaction between the electronic pair of the oxygen in the carbonyl group and the heteroatom in the neighboring ring, indicating a favored conformation. ${ }^{11-13}$ Electrochemical studies showed the existence of irreversible and asymmetrical peaks. This behavior was explained by the simultaneous oxidation of the heterocyclic rings and the functional groups acting as bridges. ${ }^{14}$ ${ }^{16}$ The reduction of the species involves only the carbonyl groups through a free radical. ${ }^{17}$ This process is very close to the reduction mechanism of chalcones proposed in Cassidy \& Whitcher ${ }^{18}$ and in Tirouflet \& Corvaisier. ${ }^{19}$<smiles>Cc1ccsc1/C=C/C(=O)/C=C/c1sccc1C</smiles>

Following our interest in this kind of compounds, mainly directed to their use as potential ligand in further complex development we have synthesized and structurally characterized a new compound in this populous family: 1,5bis(3-Methyl-2-thienyl)penta-1,4-dien-3-one, $\mathrm{C}_{15} \mathrm{H}_{14} \mathrm{OS}_{2}$ (see scheme 1)

\section{EXPERIMENTAL}

\section{SYNTHESIS}

The compound was synthesized according to Miller \& Nord. ${ }^{20}$

3-methyl-2-thiophenecarboxaldehyde $(0.0219 \mathrm{~mol})$ was mixed with acetone $(0.0267 \mathrm{~mol})$. The mixture was cooled to $0^{\circ} \mathrm{C}$ and $\mathrm{KOH} / \mathrm{Ethanol} 20 \%$ was slowly added.

The mixture was further stirred by $7 \mathrm{~h}$, it was filtered and washed with ethanol. Pale yellow crystals were obtained from an ethanol/water solution (5:1). The yield was 70\%. Elemental Analysis (\%) (exp/theo): 65.65/65.66 (C); 5.08/5.14 (H); 5.73/5.83 (O); 23.12/23.37 (S).

The IR spectrum shows the characteristic bands of the functional groups of the compound, but slightly displaced. The molecule is nearly planar and the conjugation of $\mathrm{C}=\mathrm{O}$ with a $\mathrm{C}=\mathrm{C}$ bond result in delocalization of the $\pi$ electrons in both unsaturated groups, causing absorption at lower wave-numbers; then $\mathrm{C}=\mathrm{O}, 1651 \mathrm{~cm}^{-1} ; \mathrm{C}=\mathrm{C}, 1600 \mathrm{~cm}^{-1}$.

\section{STRUCTURE DETERMINATION}

The present structure posed no problems for data collection. Hydrogen atoms were placed at calculated positions (C-H: $0.93-0.97 \AA)$ and allowed to ride; methyl groups were allowed to rotate as well. Displacement factors were taken as $\mathrm{U}(\mathrm{H}) \mathrm{isot}=\mathrm{x} \cdot \mathrm{U}($ host $), \mathrm{x}: 1.2-1.5$

\section{COMPUTING DETAILS}

Data collection: SMART-NT; ${ }^{21}$ cell refinement: SAINT-NT; ${ }^{21}$ data reduction: SAINT-NT; program(s) used to solve structure: SHELXS97:22 $\operatorname{program}(\mathrm{s})$ used to refine structure: SHELXL97; ${ }^{22}$ molecular graphics: SHELXTL-NT:23 software used to prepare material for publication: SHELXTL-NT, PLATON. ${ }^{24}$

\section{RESULT AND DISCUSSION}

Figure 1 shows an ellipsoid plot of $\mathrm{C}_{15} \mathrm{H}_{14} \mathrm{OS}_{2}$; the molecule consists of two terminals thiophene rings bridged by a penta-1,4-diene-3-one link, in such a way as to have both sulfur atoms in the rings at the same side of the bridge, the carbonyl oxygen being trans to both. The Table 1 shows some crystal data.

The overall group is almost planar, with a slight twist along the bridge axis, leading to a small rotation (16.1(1)\%) between the terminal rings. G. Liang et al. has found a similar rotation $\left(5.16(9)^{\circ}\right)$ in a similar but more rigid compound. ${ }^{8}$

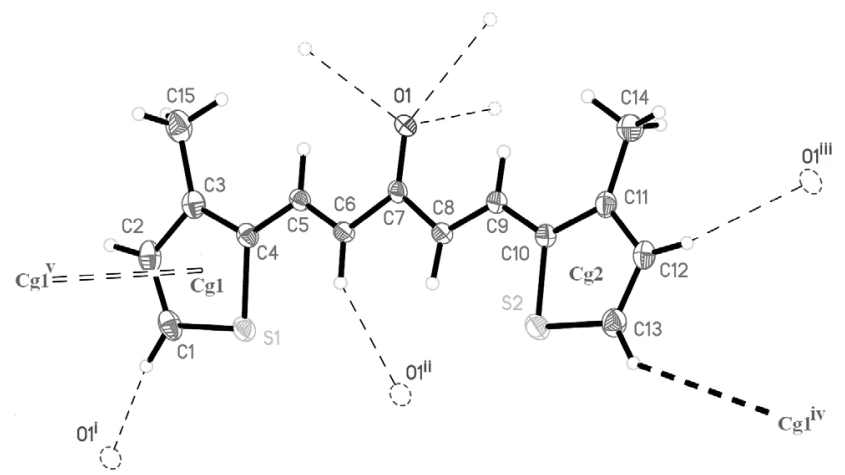

Fig. 1: Molecular diagram of $\mathrm{C}_{15} \mathrm{H}_{14} \mathrm{OS}_{2}$. Anisotropic displacement ellipsoids draw at a $40 \%$ level. H-bonding interactions with neighboring molecules presented in simple broken, $\mathrm{C}-\mathrm{H} \cdots \pi$ bonds in heavy broken, and $\pi-\pi$ bonds in double broken lines Symmetry codes, as in Tables 2 and 3. 
Table 1. Crystal data.

\begin{tabular}{|l|l|}
\hline $\mathrm{C}_{15} \mathrm{H}_{14} \mathrm{OS}_{2}$ & $V=2835.7(2) \AA^{3}$ \\
\hline$M r=274.38$ & $Z=8$ \\
\hline Orthorhombic, $P b c a$ & Mo $\mathrm{K} \alpha$ \\
\hline$a=15.8094(3) \AA$ & $\mu=0.36 \mathrm{~mm}^{-1}$ \\
\hline$b=7.7683(6) \AA$ & $T=100(2) \mathrm{K}$ \\
\hline$c=23.0896(8) \AA$ & $0.40 \times 0.18 \times 0.15 \mathrm{~mm}$ \\
\hline Data collection & \\
\hline $\begin{array}{l}\text { APEXII, Bruker-AXS } \\
\text { diffractometer }\end{array}$ & 2487 independent reflections \\
\hline $\begin{array}{l}\text { Absorption correction: multi-scan } \\
\left.\text { SADABS (Sheldrick, } 2002^{25}\right)\end{array}$ & 2119 reflections with $I>2 \sigma(I)$ \\
\hline$T$ min $=0.92, T$ max $=0.95$ & $R$ int $=0.038$ \\
\hline 12351 measured reflections & \\
\hline Refinement & 165 parameters \\
\hline$R\left[F^{2}>2 \sigma\left(F^{2}\right)\right]=0.040$ & H-atom parameters constrained \\
\hline$w R\left(F^{2}\right)=0.100$ & $\Delta \rho$ max $=0.65$ e $\AA^{-3}$ \\
\hline$S=1.05$ & $\Delta \rho$ min $=-0.34$ e $\AA^{-3}$ \\
\hline 2487 reflections & \\
\hline
\end{tabular}

Bonds along the bridge are almost theoretical, with differences between equivalent ones being smaller that their respective s.u.'s; slightly larger, though still non significant, differences appear in the thiophene rings.

In fact, in several of similar studied compounds the molecules can be considered featureless and their most appealing details should be looked at their intermolecular interactions, of varied type and strength, leading to different packing schemes. In the present case, oxygen $\mathrm{O} 1$ lies in a rather unscreened position (Fig 1), favoring its role as a triple H-bond acceptor (Fig 2) of three non-conventional C-H $\cdots \mathrm{O}$ bonds (Table 2, three uppermost entries). These interactions are completed by a C-H $\cdots \pi \mathrm{H}$-bond (Table 2, last entry) as well as $\pi-\pi$ contacts (Table 3 ) which connect evenly neighboring molecules to generate a rather compact 3D structure. Other important distances are in Table 4.

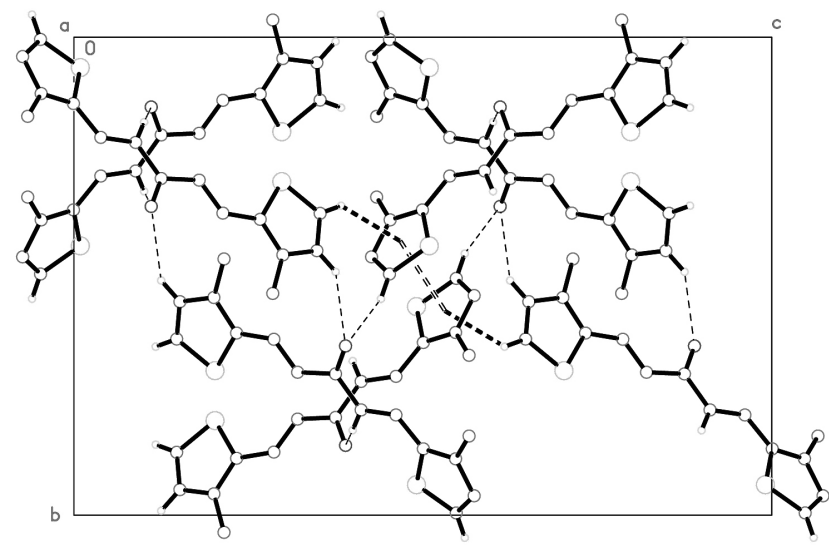

Fig. 2: Packing diagram of the H-bonded 3D structure of $\mathrm{C}_{15} \mathrm{H}_{14} \mathrm{OS}_{2}$. The interaction codes are the same as in Fig. 1
Table 2. Hydrogen-bond geometry $\left(\AA,{ }^{\circ}\right)$

\begin{tabular}{|c|c|c|c|c|}
\hline$D-\mathrm{H} \cdots A$ & $D-\mathrm{H}$ & $\mathrm{H} \cdots A$ & $D \cdots A$ & $D-\mathrm{H} \cdots A$ \\
\hline $\mathrm{C} 1-\mathrm{H} 1 \cdots \mathrm{O} 1 \mathrm{i}$ & 0.93 & 2.35 & $3.248(3)$ & 162 \\
\hline $\mathrm{C} 6-\mathrm{H} 6 \cdots \mathrm{O} 1 \mathrm{ii}$ & 0.93 & 2.42 & $3.213(3)$ & 143 \\
\hline $\mathrm{C} 12-\mathrm{H} 12 \cdots \mathrm{O} 1 \mathrm{iii}$ & 0.93 & 2.56 & $3.374(3)$ & 147 \\
\hline $\mathrm{C} 13-\mathrm{H} 13 \cdots \mathrm{Cg}$ liv & 0.93 & 2.90 & $3.683(3)$ & 141 \\
\hline
\end{tabular}

Symmetry codes: (i) $x+1 / 2,-y+1 / 2,-z$; (ii) $-x+1 / 2, y+1 / 2, z$; (iii) $-x$, $y+1 / 2,-z+1 / 2$; (iv) $-x+1 / 2,-y+1, z+1 / 2$.

Table 3. $\pi \cdots \pi$ contacts $\left(A,{ }^{\circ}\right)$ for $\mathrm{C}_{15} \mathrm{H}_{14} \mathrm{OS}_{2}$

\begin{tabular}{|l|l|l|l|l|l|}
\hline Compound & Group 1 & Group 2 & $\operatorname{ccd}(\AA)$ & $\mathrm{da}\left({ }^{\circ}\right)$ & $\operatorname{ipd}(\AA)$ \\
\hline $\mathrm{C}_{15} \mathrm{H}_{14} \mathrm{OS}_{2}$ & $C g 1$ & $C g 1 \mathrm{v}$ & $3.542(2)$ & 0.00 & $\begin{array}{l}3.43 \\
(2)\end{array}$ \\
\hline
\end{tabular}

Symmetry codes: (v) $-x,-y, 1-z ; C g 1$ : defined in Fig. 1 cc (center-to-center distance): distance between ring centroids; da: (dihedral angle) angle between planar groups; ipd (interplanar distance): mean distance from one plane to the neighbouring centroid. (For details, see Janiak 200026)

Table 4. Some important distances $(\mathcal{A})$ and angles $\left({ }^{\circ}\right)$

\begin{tabular}{|l|l|l|}
\hline C4-C5: 1,446 & C7-O1: 1,241 & C1-S1-C4: 91,35 \\
\hline C5-C6: 1,341 & C4-S1: 1,749 & C10-S2-C13: 91,69 \\
\hline C6-C7: 1,473 & C1-S1: 1,715 & C6-C7-C8: 117,16 \\
\hline C7-C8: 1,473 & C10-S2: 1,743 & C6-C7-O1: 121,50 \\
\hline C8-C9: 1,344 & C13-S2: 1,718 & C8-C7-O1: 121,34 \\
\hline C9-C10: 1,439 & & \\
\hline
\end{tabular}

\section{ACKNOWLEDGEMENTS}

We acknowledge funding by project DIUC 208.021.026-1.0 and 209.021.028-1.0. Dr. Thierry Roisnel of Centre de Diffractométrie X of Université de Rennes 1 "Sciences Chimiques de Rennes" by diffraction experiences.

We also acknowledge the Spanish Research Council (CSIC) for providing us with a free-of-charge license to the CSD system (Allen, 2002 27 ).

\section{REFERENCES}

1) Lee, C., KIm, K. J. \& Rhee, S. B. Synth. Met. 69, 295-296 (1995).

2) Diaz, F. R., del Valle, M. A. \& Brovelli, F. J. Applied Polymer Science, 89, 1614-1621(2003).

3) Nguyen, T. P., Molinie, P. \& Destruel, P. (2000). Handbook of Advanced Electronic and Photonic Materials and Devices; Nalwa, H.S., Ed; Academia: San Diego

4) Brovelli, F., Del Valle, M. A., Díaz, F. R. \& Bernede, J. C.. Bol. Soc. Chil. Quim. 46, 319 (2001a)

5) Brovelli, F., Bernede, J. C., Marsillac, S., Díaz, F. R., del Valle, M. A. \& Beaudouin, C. J. Applied Polymer Sci. 86, 1128-1137 (2002).

6) Fowler, R. H. \& Nordheim, L. Proc. R Soc Lond A, 119, 173-181 (1928).

7) Diaz, F. R., del Valle, M. A. \& Brovelli, F. J. Applied Polymer Science, 89, 1614-1621(2003)

8) G. Liang, S. Yang, X. Wang, Y. Li, X. Li. Acta. Cryst., E63, o4118-o4121 (2007).

9) Brovelli, F., Rivas, B. L. \& Basaez, L. J. Chil. Chem. Soc, 48, 135-140 (2003).

10) Y. Moreno, F. Brovelli, A. Buljan, J. Belmar. J. CHIL. CHEM. SOC., 53, 1689-1693 (2008).

11) Distefano, G., Jones, D., Guerra, M., Favaretto, I., Modelli, A. \& Mengoli, G. J. Phys. Chem., 95, 9746-9753 (1991).

12) Cheng, C. L., John, I. G., Ritchie, G. L. D., Gore, P. H. \& Farell, L. J. Chem. Soc. Perkin Trans 2, 744 (1975).

13) Benassi, R., Folli, U., Iarossi, D., Mussatti, A., Nardelli, M., Schenetti, L. \& Taddei, F. J. Chem. Soc. Perkin. Trans 2, 1851 (1987).

14) Brovelli, F., Díaz, F. R., del Valle, M. A., Bernede, J. C. \& Molinie, P. Synth. Met 122, 123-126 (2001b).

15) Onoda, M., Morita, S., Iwasa, T., Nakayama, H. \& Yoshino, K. J. Chem. Phys. 95, (11) 8584-8584 (1991). 
16) Randazzo, M., Toppare, L. \& Fernandez, J. Macromolecules, 27, 51025106 (1994).

17) Mann, C. \& Barnes, K. (1970). Electrochemical Reaction in Nonaqueous System, Marcel Dekker, New York

18) Cassidy, J. E. \& Whitcher, W. J. J. Phys. Chem 63, 1824-1826 (1959).

19) Tirouflet, J. \& Corvaisier, A. Bull. Soc. Chim. France 99, 535 (1962).

20) Miller, R. \& Nord, F. J. Org. Chem. 16, 1720 (1951).

21) Bruker (2006). SMART, SAINT. Bruker AXS Inc., Madison, Wisconsin, USA.
22) Sheldrick, G. M. (1997). SHELXS97 and SHELXL97. University of Gottingen, Germany.

23) Bruker (2001). SHELXTL-NT. Bruker AXS Inc., Madison, Wisconsin, USA.

24) Spek, A. L. J. Appl. Cryst. 36, 7--13 (2003)..

25) Sheldrick, G. M. (2002). SADABS. Bruker AXS Inc., Madison, Wisconsin, USA.

26) Janiak, C. J. Chem. Soc., Dalton Trans., 3885-3898 (2000).

27) Allen, F. H. Acta Cryst. B58, 380-388 (2002). 